\title{
A Review on Effect of Process Parameters on Machining Characteristics of Rotary Ultrasonic Machine
}

\author{
Vikas Singh, Dheeraj Joshi, Praveen Saraswat \\ Department of Mechanical Engineering, Swami Keshvanand Institute of Technology, Management and \\ Gramothan, Jaipur-302017 (INDIA) \\ Email-praveenmeskit@gmail.com
}

Received 27.07.2020 received in revised form 31.07.2020, accepted 06.10.2020

\begin{abstract}
Ultrasonic machining (USM) is a process used for machining material with high hardness and brittle nature that are difficult to machine. The USM has an upper hand on the other non-conventional process like electron discharge and laser beam machining which have thermal effect on workpiece. Workpiece chracteristics are also not limited by chemical and electrical properties. This paper discusses about the rotary ultrasonic drilling (RUD) machining process which is a variant of USM and the recent developments that it gone under through the RUD. This paper also reviews about the various methodology adopted by researchers to achieve optimum output parameters like Material Removal Rate, Surface roughness, Edge chipping etc. by altering the process characteristics like spindle speed, feed rate, ultrasonic power etc. as well as machine characteristics like tool type, support length etc. The paper also deals with the material used with the RUD worldwide and the findings of researchers on them.
\end{abstract}

Keywords:Hybrid machining, Rotary ultrasonic drilling, Brittle material, Edge chipping

\section{INTRODUCTION}

Modernization demands use of advance materials to take into account its various needs of mankind.These matertials may include composites, ceramics and superalloys etc. thatare difficult to machine and process. Various developments has been made by researchers to resolve this problem, one of such effective development was to utilize ultrasonic vibration in machining. It was found that the better option to machine a hard (harder than HRC 40) and brittle material is ultrasonic one. Ultrasonic Machining is efficient one as it processes the material irrespective of its chemical and electric behavior. Rotary ultrasonic machining (RUM) is the advancement in USM which incorporates hybrid process i.e. Rotary assisted grinding and ultrasonic vibration. Unlike the conventional grinding RUD gives better surface finish, increased MRR, reduction in edge chipping and surface parameter like burr height, tearing defect etc.
Rotary ultrasonic machine convert the low frequency electric signals into higher one which are fed into transducer to convert them into mechanical to and fro motion get transmitted to tool through horn. This vibratory motion generally occur at a high frequency $(>20 \mathrm{kHz})$ along the tool feed direction. A coolant system ensures the heat dissipation at cutting zone. The material is removed by the combine action of hammering, extraction and erosion by the diamond coated tool abrasives. Fig. 1 illustrates the basic component of Rotary ultrasonic machine.

\section{BASIC COMPONENTS OF ROTARY ULTRASONIC MACHINE}

There are different variety of Rotary ultrasonic machine employed in industries based on their capabilities, Power rating, table size etc. but these all shares some basic component as mentioned below:

1. Transducer- It is used to convert electrical energy to mechanical vibration.

2. Horn - It is used to amplify the mechanical vibration and act as a connector between transducer and tool to transmit vibration.

3. Tool - Tools of different types with or without abrasive are utilized for material removal.

4. Motors-These are of two types. An axis motor is used to give axial motion while spindle motor provides rotation to tool.

5. Work Table - This provides the area on which RUM can work on, this is what you clamp your work onto.

6. Coolant Tank- It houses coolant pump to circulate coolant in the machine through coolant nozzle.

7. Control Panel - It houses various electrical and electronic part of control machine along with control PC, monitoring screen and ultrasonic generator. 


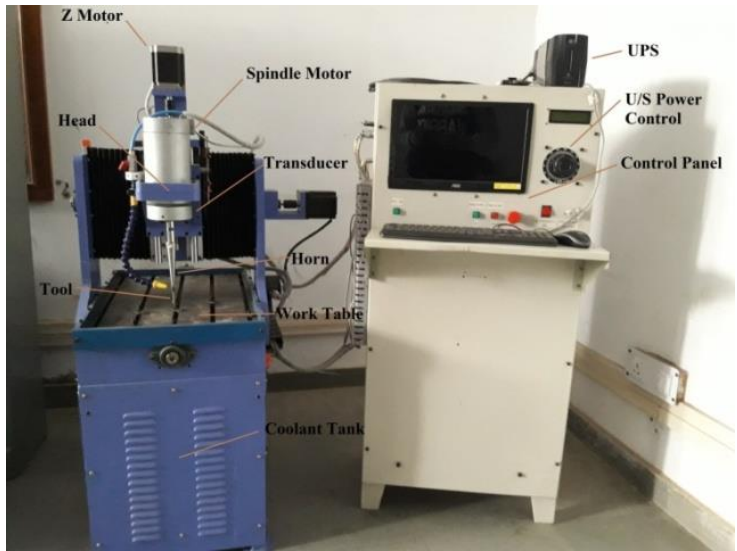

Figure 1: Basic Components of Rotary Ultrasonic Machine

\section{METARIAL REMOVAL MECHANISM}

As mentioned earlier, RUD is the combination of two kind of processes utilized in machining i.e. USM and grinding. Figure 2 illustrates the material removal mechanism of rotary ultrasonic machine. The material removal mechanisms include:

- abrasion action caused by pure rotational movement of tool,

- hammering action caused by ultrasonic movement and

- extraction motion caused by combine action of ultrasonic vibration and rotational movement.

Figure 2 illustrate the material removal mechanism of Rotary ultrasonic machine.

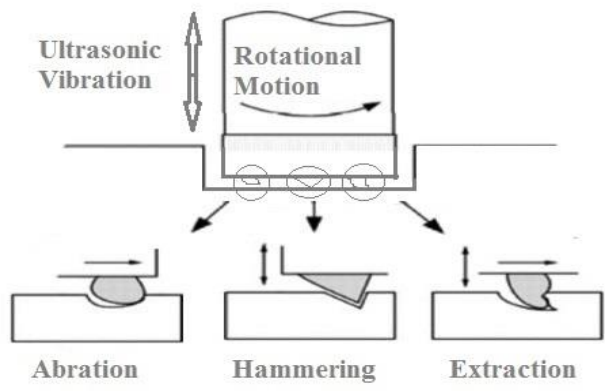

Figure 2: Material Removal Mechanism

\section{PROCESS PARAMETERS AND THEIR EFFECT}

In this section, we review the research findings on process parameters and their effect on response variables experimentally determined by various researchers. Further, the discussion has been catergorically mentioned as mentioned below:

\subsection{Effect on Edge chipping}

The lower amount of edge chipping is seen when rotational speed of tool is kept higher along with higher ultrasonic power and lower feed rate [1]. The dependency of edge chipping on ultrasonic amplitude is weaker while edge chipping increases with increase in feed rate [2].A reduction in edge chipping can also be achieved by utilizing conical drills. At a particular feed rate the characteristics angle of conical drill have to exceed a critical value. The higher critical characteristics angle is needed for higher feed rate [3].

An experiment conducted on quartz glass on RUM shows roughly $60 \%$ reduction in edge chipping by utilizing the step drill without having negative influence on the tool's life. To claim the effectiveness of utilizing step drill, the thickness of step drill should not be greater than that of drill end face and the height of step should exceed a critical value [4].Size of edge chipping also has a direct correlation with the undrilled thickness. The larger lateral crack developed in RUM which provide shielding effect on the growth of radial and medial cracks which are mainly responsible for edge chipping [5].An experimental study on alumina ceramics on RUM shows that ultrasonic power and coolant pressure has insignificant effect on edge chipping reduction [6].

A Comparison with the conventional ultrasonic drilling of quartz glass with RUM shows that RUM can reduce the edge chipping size at the exit side of hole by $45 \%$ [7]. An experimental investigation done on K9 glass shows that intermittent RUM can lead to reduction in the size of edge chipping while comparing with continuous RUM. This relationship is close to linear [8]. Shape of the tool also plays a significant role in reducing the edge chipping size. An investigation of tool shape has been done on edge chipping in RUM and it is seen that the tool face surface's slope if modified from 0 to 30 degree, cause a drop in the value of stress peak up to $55 \%$ thus lowering the size of edge chipping [9]. An experiment was performed on quartz glass under RUM which reveals that when a compound tool (i.e. Taper tool and Step tool) is used than the size of edge chipping get reduced by $60-80 \%$ [10]. Material removal process of individual diamond abrasives causes edge chipping at the entrance side of hole while the effect of macro force of all the diamond abrasives causes edge chipping at the exit of hole [11].

An experimental investigation performed on Alumina ceramic suggest that support length can help to lower the size of edge chipping by roughly $50 \%$ [12].Concave circular tool is found to be the best out of pin pointed conical tool, Flat pointed cylindrical tool, hollow abrasive tool and concave circular tool while operating float glass under RUM especially to achieve minimum chip radial distance during rotary ultrasonic drilling [13]. 


\subsection{Effect on Material Removal Rate}

It has been observed that hollow tool significantly improves the material removal rate as compared to solid tool. The MRR obtained by hollow tool is roughly two times higher than the obtained by solid tool [14]. The MRR obtained in RUM is higher while comparing with conventional grinding [15]. The material removal in RUM is mainly brittle fracture with slighter plastic deformation in rotary ultrasonic machining of quartz ceramic [16].

An experiment conducted on BK7 optical glass under which the feed rate is observed as the most influencing operating parameter while dealing with material removal rate as a little increase in it sharply increases the MRR [17]. The feed rate of tool is found as the most critical process parameter for MRR [18].

At the low value of feed rate and elevated value of spindle speed and ultrasonic power mixed flow of the material is seen in BK7 glass [19]. The Scanning Electron Microscope (SEM) micrograph of machined surface of BK7 glass under RUM shows the presence of plastically deformed region at low feed rate while at higher feed rate values dominance of brittle fracture can be seen [20].

\subsection{Effect on Surface Integrity}

\subsubsection{Delamination}

A comparison with the conventional ultrasonic drilling shows that delamination at entry and exit of drilled hole is prevented along with improved circumferential edge quality of hole in rotary ultrasonic drilling [14].

Lesser amount of delamination is achieved by a tool which has larger grain size while conducting experiment on lower fiber volume composites. Additionally it is found that high feed rate and lower spindle speed induces more amount of delamination [21].

\subsubsection{Geometrical Error}

The diameter of hole obtained in RUM while comparing with conventional grinding is higher [15]. The quality at the exit of drilled hole is mainly determined by drilling force [22].

It is found that both cutting speed and direction of fiber cutting significantly affect the surface topography in RUM of $\mathrm{C} / \mathrm{Sic}$ composites. The relatively low amount of spindle speed and high amount of ultrasonic amplitude apparently plays an important role in further improvement of hole surface quality [23].

The broken and discontinuous chips get formed by the intermittent movement of tool in feed direction due to which built up edge (BUE) get lowered and an improved quality of hole is obtained [24]. (a) Cylindricity and Conicity

An investigation on titanium alloy revealed that the rotational speed and diameter of tool has considerable effect on cylindricity of hole. The interaction of the diameter of tool with cutting speed and feed rate with ultrasonic power also has a noticeable effect on cylindicity [25]. An experiment performed on Inconel 718(Nickel alloy) concluded that high feed rate results into greater compressive stresses around the workpiece as well as tool. This degrades the quality index like conicity and circularity. Thus to achieve lowest conicity a combination of high spindle speed and low feed ate is desired [26].

An experiment conducted on RUM of titanium alloy (Ti6Al4V) reveals that cylindricity error increases with the increase in ultrasonic power while the effect of feed rate on cylindicity error depends on the levels of spindle speed and tool diameter [27]. An elevated feed rate of tool degrades the quality of machined hole by increasing the size of taper and chipping width. Combination of high ultrasonic power along with lower feed and spindle speed results to improve the surface finish. This improvement in surface roughness was due to mixed flow of material at these selected levels of input process parameters [28].

\section{(b) Overcut}

An investigation on Titanium alloy revealed that the rotational speed of tool and diameter of tool has considerable effect on the overcut [25]. An experimental study on RUM of Titanium alloy (Ti6Al4V) suggests that the overcut error typically reduces with the increase in both i.e. Spindle speed of tool and diameter of tool [27].

\subsubsection{Tearing Defect}

While conducting an experiment on fiber reinforced ceramic matrix composite on rotary ultrasonic machining it was found that a large thrust force corresponds to a larger drill displacement in the course of formation of tearing defect. Tearing defect can be reduced by $60 \%$ at exit side of hole under RUM. The further reduction in tearing defect can be achieved by increasing the level of spindle speed and ultrasonic vibrational amplitude of the tool while decreasing the feed rate [29].

At the entrance of hole, the tearing defect is due to material removal mechanism of individual diamond abrasives while at hole exit the tearing defect was found due to the effect of macro force of all the diamond abrasives [11].

\subsubsection{Exit Burr}

An experiment conducted in cryogenic environment on CFRP under RUM shows that the RUM in the cryogenic environment is superior as it 
produces a lower exit burr area and average roughness of machined surface. Further minimum exit burr area is obtained we decrease spindle speed along with increment in feed rate and ultrasonic power. The significance of ultrasonic power is high when reducing burrs at exit with the contribution of approx. $52.45 \%$. The significance on producing a better surface finish for drilled hole noted for the spindle speed is $30.88 \%$ and that for feed rate is $30.83 \%$ which is almost equal [30].

\subsection{Effect on Surface Roughness}

The surface roughness of the drilled hole obtained in RUM is lower while comparing with conventional grinding [15].

AComparison with the conventional ultrasonic drilling of quartz glass with RUM shows that RUM can enhance the surface quality of drilled hole by reducing the roughness of surface, especially when the machine is operating at relatively lower spindle speed [7]. It is observed that surface finish get deteriorated when feed rate is increased. The feed rate has highest contribution i.e. $76.19 \%$ on roughness of surface while the contribution of the spindle speed is $5.48 \%$ and of ultrasonic power is $8.81 \%$ [19].

The cross linking of cracks cause pullout of the grains thus deeper groves get generated on machined surface. The surface roughness of machined face increases sharply with increase in the tool feed rate while surface roughness decreases with increase in rotational speed of tool and ultrasonic power [31].

\subsection{Effect on Tool Wear}

The optimum parameters for lesser tool wear are low feed rate, high cutting speed and adequate amount of axial vibration [32]. Severe tool wear is seen in the initial stage of experimentation on BK7 optical glass due to grain fracture and bond fracture [17].

An experiment performed on titanium alloy (Ti6Al4V) resulted that at high spindle rotation and larger diameter of tool with an increment either in feed rate or ultrasonic power can bring a very sharp increment in tool wear [27]. The minimum amount of wear is seen on the tool that contains minimum area of surface (both end and lateral face) in contact with the workpiece [33].

\subsection{Effect on Cutting Force}

Lower cutting force is desired as it provides reduction in edge chipping. An experiment is conducted on sapphire and it is observed that lower cutting force is achieved by increasing spindle speed and ultrasonic power while reducing the feed rate supplied to tool [34]. Feed rate and spindle speed two prime process parameters which affect the cutting force significantly [2].

An experiment conducted on carbon fiber reinforced plastic (CFRP) shows that the most significant process parameter is feed rate followed by rotation speed of tool, size of abrasive, amplitude of vibration and concentration of abrasive. As vibration amplitude, rotational speed of the tool and size of abrasive increases, cutting force decreases while it increases as feed rate and abrasive concentration increases [35].

In RUM cutting force is less while comparing with conventional grinding at higher rotational speed and feed rate [15]. Rotary ultrasonic drilling can drastically reduce the cutting force $(15.1 \%$ $22.5 \%$ ) as compared with conventional drilling [22]. A Comparison with the conventional ultrasonic drilling of quartz glass with RUM shows that $55 \%$ reduction in cutting force is achieved under RUM on an average [7].

An experimental investigation done on $\mathrm{K} 9$ glass shows that intermittent RUM can lead to reduction in the cutting force while comparing with continuous RUM and had a relationship close to linear [8]. An experiment performed on titanium alloy (Ti6Al4V) resulted that the cutting force can be reduced when we increase ultrasonic power at the given condition of low spindle speed and diameter of tool [27].

\subsection{Effect on Thrust Force}

It is experimentally founded that harmonic movement of the drill bit has an influence over the built up edge (BUE) formation which affect the thrust force. A comparison of RUM with conventional drilling showed that reduction in BUE is achieved in RUM as the contact time between drill bit and workpiecedecrease [36].

\subsection{Effect on Vibration amplitude}

A mechanist model was developed to obtain amplitude of vibration by Cutting force. It is seen that amplitude of ultrasonic vibration increases when the ultrasonic power and feed rate increases while rotational speed of tool decreases [37].

\section{CONCLUSION}

The above literature establishes the importance of RUM for machining brittle and hard material. The recent developments shows that

1. The reduction in the edge chipping size can be achieved at higher rotational speed and ultrasonic power supplied to the tool along with lowering the feed rate. It is verified that reducing the cutting force helps in smaller edge chipping. The results obtained after the experiment show that roughly $60 \%$ reduction 
in the edge chipping can be obtained by utilizing the step drill while the compound tool (Taper with step) can reduce it by $60-80 \%$. Undrilled thickness and support length also have influence over size of edge chipping as increment in these can reduce edge chipping size. Reduction of about $50 \%$ can be achieved by increasing support length.

2. A high MRR can be obtained by increasing the feed rate of tool as it is most influencing parameter. A hollow tool used in RUM can provide roughly two times higher MRR as given by solid tool. A mixed flow of material is seen at low feed rate and higher spindle speed and ultrasonic power.

3. Feed rate has high contribution $(76.19 \%)$ to surface roughness than ultrasonic power $(8.81 \%)$ and tool rotational speed (5.48). The low value of surface roughness is achieved when we decrease feed rate while increasing spindle speed and ultrasonic power to tool.

4. Tool wear is undesirable as well as unavoidable. Thus adequate amount of axial vibration along with low feed rate and high cutting speed and tool diameter can reduce the amount of tool wear significantly.

5. It has been noticed that the delamination is reduced we use tool with larger grain size along with low feed rate and high rotational speed. High rotational speed and bigger diameter of tool also helps in reducing overcut error while low ultrasonic power and feed rate can improve conicity and cylindricity error.

6. The intermittent action of RUM tool provide less contact time of tool with workpiece thus helps in reducing the Built up Edge formation which ultimately results in lower thrust force and better surface quality.

7. Tearing defect can be minimized by elevating the ultrasonic vibrational amplitude and tool rotational speed while cutting the feed rate. It is observed that $60 \%$ reduction in tearing defect occurs under RUM when compared with USM.

8. Ultrasonic power has significant effect over exit burr. Thus exit burr area can be reduced by increasing the ultrasonic power and feed rate while reducing spindle speed spatially in cryogenic environment.

9. The most significant input variable for reducing the cutting force is feed rate after which tool rotation speed and abrasive size have their significance, vibration amplitude and abrasive concentration have lowest significance among all input variables. The value of cutting force decrease as amplitude of vibration, rotation speeds of tool and size of abrasive particle increase and feed rate along with concentration of abrasive decrease. Rotary ultrasonic drilling can drastically reduce the drilling force (15.1\%-22.5\%) compared with conventional drilling and while comparing with conventional USM it can reduce cutting force by $55 \%$.

\section{REFERENCES}

[1] Cong W. L., Feng Q., Pei Z. J., Deines T. W. \& Treadwell C. (2012). Edge chipping in rotary ultrasonic machining of silicon. International Journal of Manufacturing Research, 7(3), 311-329.

[2] Yuan S., Zhang C., Amin M., Fan H. \& Liu M. (2015) Development of a cutting force prediction model based on brittle fracture for carbon fiber reinforced polymers for rotary ultrasonic drilling. The International Journal of Advanced Manufacturing Technology, 81(5-8), 1223 1231.

[3] Wang J., Feng P. \& Zhang J. (2016). Investigations on the edge-chipping reduction in rotary ultrasonic machining using a conical drill. Proceedings of the Institution of Mechanical Engineers, Part B: Journal of Engineering Manufacture, 230(7), 1254-1263.

[4] Wang J., Feng P. \& Zhang J. (2016). Reduction of edge chipping in rotary ultrasonic machining by using step drill: a feasibility study. The International Journal of Advanced Manufacturing Technology, 87(9-12), 28092819.

[5] Wang J., Zha H., Feng P. \& Zhang J. (2016). On the mechanism of edge chipping reduction in rotary ultrasonic drilling: A novel experimental method. Precision Engineering, 44, 231-235.

[6] Singh R. P. \& Singhal S. (2017). Investigation of machining characteristics in rotary ultrasonic machining of alumina ceramic. Materials and Manufacturing Processes, 32(3), 309-326.

[7] Wang J., Zhang J., Feng P., Guo P. \& Zhang Q. (2018). Feasibility study of longitudinal-torsional-coupled rotary ultrasonic machining of brittle material. Journal of Manufacturing Science and Engineering, 140(5), 051008 .

[8] Fernando P., Zhang M., Pei Z. \& Cong W. (2017). Intermittent and continuous rotary ultrasonic machining of $\mathrm{K} 9$ glass: an experimental investigation. Journal of Manufacturing and Materials Processing, 1(2), 20.

[9] Kolíková L., Rolník L. \& Duriš R. (2019). Investigation of vibration effects and tool shape on edge chipping phenomenon occurring during rotary ultrasonic drilling. Journal of Sound and Vibration, 439, 251-259.

[10] Wang J., Feng P. \& Zhang J. (2018). Reducing edge chipping defect in rotary ultrasonic machining of optical glass by compound step-taper tool. Journal of Manufacturing Processes, 32, 213-221.

[11] Wang J., Zhang J., Feng P. \& Guo P. (2018). Damage formation and suppression in rotary ultrasonic machining of hard and brittle materials: a critical review. Ceramics International, 44(2), 1227-1239.

[12] Popli D. \& Gupta M. (2018). A chipping reduction approach in rotary ultrasonic machining of advance ceramic. Materials Today: Proceedings, 5(2), 6329-6338.

[13] Sharma A., Jain V. \& Gupta D. (2019). Comparative analysis of chipping mechanics of float glass during rotary ultrasonic drilling and conventional drilling: For multi-shaped tools. Machining Science and Technology, $1-22$.

[14] Debnath K., Singh I. \& Dvivedi A. (2015). Rotary mode ultrasonic drilling of glass fiber-reinforced epoxy laminates. Journal of Composite Materials, 49(8), 949963. 
[15] Ning F. D., Cong W. L., Pei Z. J. \& Treadwell C. (2016). Rotary ultrasonic machining of CFRP: a comparison with grinding. Ultrasonics, 66, 125-132.

[16] Singh R. P. \& Singhal S. (2018). Experimental investigation of machining characteristics in rotary ultrasonic machining of quartz ceramic. Proceedings of the Institution of Mechanical Engineers, Part L: Journal of Materials: Design and Applications, 232(10), 870889.

[17] Kumar V. \& Singh H. (2018). Machining optimization in rotary ultrasonic drilling of $\mathrm{BK}-7$ through response surface methodology using desirability approach. Journal of the Brazilian Society of Mechanical Sciences and Engineering, 40(2), 83.

[18] Sindhu D., Thakur L. \& Chandna P. (2018). Multiobjective optimization of rotary ultrasonic machining parameters for quartz glass using Taguchi-Grey relational analysis (GRA). Silicon, 1-12

[19] Kumar V. \& Singh H. (2018). Regression analysis of surface roughness and micro-structural study in rotary ultrasonic drilling of BK7. Ceramics International, 44(14), 16819-16827.

[20] Kumar V. \& Singh H. (2019). Optimization of rotary ultrasonic drilling of optical glass using Taguchi method and utility approach. Engineering Science and Technology, an International Journal.

[21] Baraheni M. \& Amini S. (2018). Feasibility study of delamination in rotary ultrasonic-assisted drilling of glass fiber reinforced plastics. Journal of Reinforced Plastics and Composites, 37(1), 3-12.

[22] Yuan S. M. \& Wu Q. (2016). Experimental Investigation and Optimization in Rotary Ultrasonic Drilling of $\mathrm{C} / \mathrm{C}$ Composites. In Materials Science Forum (Vol. 874, pp. 313-319). Trans Tech Publications.

[23] Wang J., Zhang J. \& Feng P. (2017). Effects of tool vibration on fiber fracture in rotary ultrasonic machining of $\mathrm{C} / \mathrm{SiC}$ ceramic matrix composites. Composites Part B: Engineering, 129, 233-242.

[24] Paktinat H. \& Amini S. (2018). Experiments and finite element simulation of ultrasonic assisted drilling. Journal of Manufacturing Science and Engineering, 140(10), 101002.

[25] Nasr M., Anwar S., El-Tamimi A. \& Pervaiz S. (2018, April). Minimization of the hole overcut and cylindricity errors during rotary ultrasonic drilling of Ti-6Al-4V. In IOP Conference Series: Materials Science and Engineering (Vol. 346, No. 1, p. 012059). IOP Publishing.

[26] Popli D. \& Gupta M. (2019). Investigation of the Circularity and Conicity of Super Alloy during Rotary Ultrasonic Machining. Iranian Journal of Science and
Technology, Transactions of Mechanical Engineering, 43(1), 809-829.

[27] Anwar S., Nasr M. M., Al-Ahmari A., Alkahtani M., Abdo B., El-Tamimi A. \& Darwish S. (2018). Rotary ultrasonic drilling of Ti6Al4V: Effects of machining parameters and tool diameter. Advances in Mechanical Engineering, 10(1), 1687814017750784.

[28] Kumar V. \& Singh H. (2019). Rotary ultrasonic Drilling of Silica Glass BK-7: microstructural investigation and process optimization through TOPSIS. Silicon, 11(1), 471-485.

[29] Feng P., Wang J., Zhang J. \& Zheng J. (2017). Drilling induced tearing defects in rotary ultrasonic machining of $\mathrm{C} / \mathrm{SiC}$ composites. Ceramics International, 43(1), 791799.

[30] Kumaran S. T., Ko T. J., Li C., Yu Z. \& Uthayakumar M. (2017). Rotary ultrasonic machining of woven CFRP composite in a cryogenic environment. Journal of Alloys and Compounds, 698, 984-993.

[31] Sindhu D., Thakur L. \& Chandna P. (2019). Parameter Optimization of Rotary Ultrasonic Machining on Quartz Glass Using Response Surface Methodology (RSM). Springer Nature B.V.

[32] Liu J. W., Baek D. K. \&Ko T. J. (2014). Chipping minimization in drilling ceramic materials with rotary ultrasonic machining. The International Journal of Advanced Manufacturing Technology, 72(9-12), 15271535.

[33] Sharma A., Jain V. \& Gupta D. (2018). Characterization of chipping and tool wear during drilling of float glass using rotary ultrasonic machining. Measurement, 128, 254-263.

[34] Zhang C. L., Feng P. F., Pei Z. J. \& Cong W. L. (2014). Rotary ultrasonic machining of sapphire: feasibility study and designed experiments. In Key Engineering Materials (Vol. 589, pp. 523-528). Trans Tech Publications.

[35] Ning F. \& Cong W. (2015, September). Rotary ultrasonic machining of CFRP: design of experiment with a cutting force model. In ASME 2015 international manufacturing science and engineering conference. American Society of Mechanical Engineers Digital Collection.

[36] Lotfi M. \& Amini S. (2017). Experimental and numerical study of ultrasonically-assisted drilling. Ultrasonics, 75, 185-193.

[37] Ning F., Wang H., Cong W. \& Fernando P. K. S. C. (2017). A mechanistic ultrasonic vibration amplitude model during rotary ultrasonic machining of CFRP composites. Ultrasonics, 76, 44-51. 